\title{
A Case of Atypical Presentation of Ovarian Torsion
}

Dr. Eldho Sajeev ${ }^{1 *}$, Dr. Riya Deshmukh ${ }^{1}$, Dr. Manpreet Singh ${ }^{1}$, Dr. M. Prabakaran ${ }^{1}$

\begin{tabular}{|c|c|}
\hline $\begin{array}{l}\text { ost graduate } \\
\text { dia }\end{array}$ & \\
\hline $\begin{array}{l}\text { Article History } \\
\text { Received: } 20.09 .2020 \\
\text { Accepted: } 12.10 .2020 \\
\text { Published: } 21.10 .2020\end{array}$ & $\begin{array}{l}\text { Abstract: Ovarian torsion is the fifth most common gynecological emergency in the world. } \\
\text { The numerous imaging characteristics and nonspecific symptoms of ovarian torsion can lead } \\
\text { to a delay in detection, with misdiagnosis being prevalent. To avoid irreversible ovarian } \\
\text { damage, early detection and restoration of blood flow are essential. For timely intervention, } \\
\text { however, increased knowledge and suspicion of this condition is needed. }\end{array}$ \\
\hline & Keywords: Ovary, Torsion, Gynecology, Necrosis. \\
\hline
\end{tabular}
https://www.easpublisher.com/easjrit

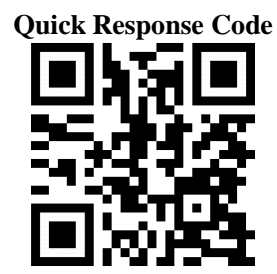

Copyright (9) 2020 The Author(s): This is an open-access article distributed under the terms of the Creative Commons Attribution 4.0 International License (CC BY-NC 4.0) which permits unrestricted use, distribution, and reproduction in any medium for non-commercial use provided the original author and source are credited.

\section{INTRODUCTION}

The twisting of an ovary on its ligamentous supports is ovarian torsion and may result in a compromised supply of blood. A word that involves either the ovary, fallopian tube, or both is adnexal torsion. It has been shown that concurrent ovarian and tubal torsion occurs in up to 67 percent of adnexal torsion cases [1]. Ovarian torsion can occur in women of all ages; however, women havethe highest incidence in their reproductive years, with pregnant women occurring in $17 \%$ to $20 \%$ of cases. This is possibly attributable to the increased prevalence at the extremes of age of physiological and pathological ovarian masses, infertility care, and pregnancy relative to that of females. Ovarian torsion signs are often nonspecific, making it difficult to distinguish between acute abdominal pain and other causes. Sharp, localised right or left lower abdominal pain and tenderness with palpable abdominal mass and peritoneal signs are included in the classic presentation.Nausea and vomiting waves as well as pyrexia were observed [2]. Patients experience eintermittent discomfort in some cases, making the diagnosis much more difficult. Here we present an atypical presentation of ovarian torsion with necrosis in a 37 year old female.

\section{Case Description}

A 37 year old female presented with c/o pain over left lower abdomen since 20 days. She had fever along with pain which was gradually progressive initially which persisted for a week. Fever subsided after 2 weeks but mild pain persisted. O/e - tenderness noted over left lower abdomen without guarding or rigidity. Hepatomegaly noted. Leukocyte count elevated. Ultrasound abdomen study showed hepatosplenomegaly, para aortic lymph nodes noted in the post renal segment of aorta on the left side. Left ovary appears enlarged with multiple air foci and central hyoechoic region. It showed increased surrounding vascularity and fat stranding. She was referred for a CT abdomen study which showed enlarged heterogenously hypodense left ovary with multiple foci of air within and associated fat stranding features suggestive of ovarian torsion with necrosis (Fig-1 Red arrows). 


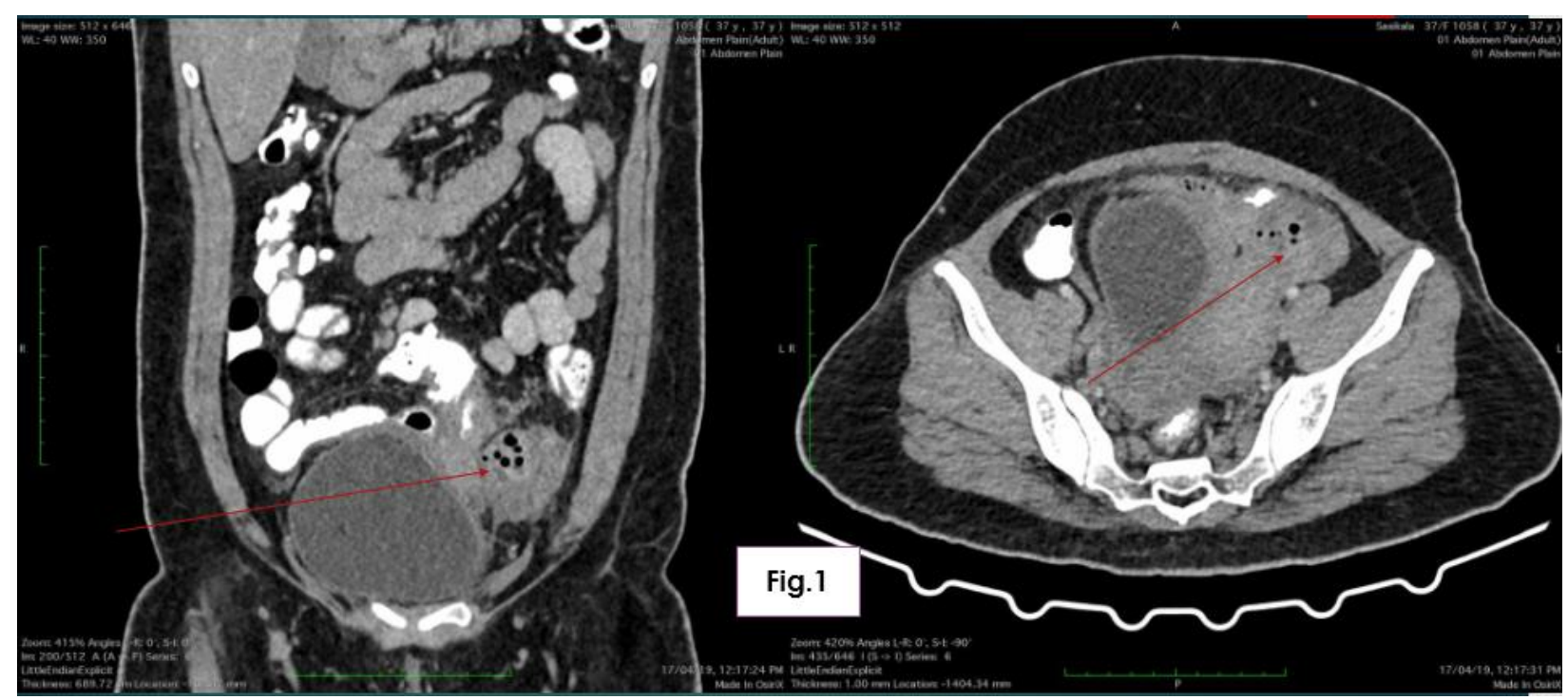

Fig-1: CECT study of abdomen shows enlarged heterogenously enhancing hypodense left ovary (Red arrow).with multiple foci of air within and associated surrounding fat stranding - features suggestive of ovarian torsion with necrosis

\section{DisCUSSION}

Ovarian torsion is the fifth most common gynecologic surgical emergency. The varied imaging features and nonspecific symptoms of ovarian torsion can lead to a delay in identification, with misdiagnosis being common. Early recognition and restoration of blood flow are important to avoid irreversible ovarian damage [3]. However, heightened awareness and suspicion of this condition are needed for timely intervention. The main feature of torsion is ovarian enlargement due to venous/lymphatic engorgement, edema and hemorrhage [4]. Secondary signs include free pelvic fluid, an underlying ovarian lesion, reduced or absent vascularity and a twisted dilated tubular structure corresponding to the vascular pedicle. An enlarged edematouos ovary with peripherally displaced follicles and reduced vascularity are the typical findings [5]. The case here presented at a late stage with necrosis. The patient was advised to undergo surgery but wasn't complying and was managed conservatively.

Conflict of interest: Author declares that they have no conflict of interest.

Ethical approval (animals): This article does not contain any studies with animals performed by any of the author(s)

\section{Authors' Contributions}

1. Dr. Eldho Sajeev (ES): Substantial contributions to the conception or design of the work; or the acquisition, analysis, or interpretation of data for the work - MP, ES, RD, MS.
2. Dr. Riya Deshmukh (RD): Drafting the work or revising it critically for important intellectual content - ES, RD, MS, MP.

3. Dr. Manpreet Singh (MS): Final approval of the version to be published - ES, RD, MS, MP.

4. Dr. M. Prabakaran (MP): Agreement to be accountable for all aspects of the work in ensuring that questions related to the accuracy or integrity of any part of the work are appropriately investigated and resolved - ES, RD, MS, MP.

\section{REFERENCES}

1. McWilliams, G. D., Hill, M. J., \& Dietrich III, C. S. (2008). Gynecologic emergencies. Surgical Clinics of North America, 88(2), 265-283.

2. Muolokwu, E., Sanchez, J., Bercaw, J. L., SangiHaghpeykar, H., Banszek, T., Brandt, M. L., \& Dietrich, J. E. (2011). The incidence and surgical management of paratubal cysts in a pediatric and adolescent population. Journal of pediatric surgery, 46(11), 2161-2163.

3. Huchon, C., \& Fauconnier, A. (2010). Adnexal torsion: a literature review. European Journal of Obstetrics \& Gynecology and Reproductive Biology, 150(1), 8-12.

4. Ding, D. C., Hsu, S., \& Kao, S. P. (2007). Isolated torsion of the hydrosalpinx in a postmenopausal woman. JSLS: Journal of the Society of Laparoendoscopic Surgeons, 11(2), 252.

5. Antoniou, N., Varras, M., Akrivis, C., Kitsiou, E., Stefanaki, S., \& Salamalekis, E. (2004). Isolated torsion of the fallopian tube: a case report and review of the literature. Clinical and experimental obstetrics \& gynecology, 31(3), 235-238. 